\title{
Análise do perfil epidemiológico em saúde Bucal de regiões distintas do Município de Penedo-AL: Estratégia de fortalecimento e redirecionamento da política de saúde
}

\section{bucal}

\author{
Analysis of the epidemiological profile in oral health in different regions of the Municipality of \\ Penedo-AL: Strategy for strengthening and redirecting oral health policy
}

Análisis del perfil epidemiológico en salud bucal en diferentes regiones del Municipio de Penedo -

AL: Estrategia de fortalecimiento y reorientación de la política de salud bucal

Recebido: 23/04/2021 | Revisado: 01/05/2021 | Aceito: 18/05/2021 | Publicado: 04/06/2021

Edilaine Soares dos Santos
ORCID: https://orcid.org/0000-0003-4748-3188
São Leopoldo Mandic, Brasil
E-mail: edilaine_ssoares@ hotmail.com
Kamila Rosamiglia Kantovitz
ORCID: https://orcid.org/0000-0003-2045-7924
São Leopoldo Mandic, Brasil
E-mail: kamilak@yahoo.com.br
Karen Dallla Costa
São Leopoldo Mandic, Brasil
E-mail: kldallacosta@ hotmail.com
ORCID: https://orcid.org/0000-0001-8539-5336
Karini Vieira Menezes de Omena
ORCID: https://orcid.org/0000-0002-9226-7743
Eniversidade Estadual de Ciências da Saúde de Alagoas, Brasil
E-mail: karinimenezes@yahoo.com.br
Charlles Petterson Andrade de Omena
ORCID: https://orcid.org/0000-0002-5693-1504
Faculdade da Cidade de Maceió, Brasil
E-mail: charllesomena@yahoo.com.br
Camylla Ataide Rezende de Gouveia
ORCID: https://orcid.org/0000-0002-6912-5317
Wisdom of Christ University, EUA
E-mail: camylla.ar@ @otmail.com

\begin{abstract}
Resumo
A realização de levantamentos epidemiológicos consitui-se de importante ferramenta para o conhecimento da prevalência das doenças bucais e, assim poder orientar o planejamento, execução e avaliação de ações de saúde em uma população. Dessa forma, foi realizado um estudo transversal com 13.706 pessoas, residentes na zona rural e na zona urbana, em faixas etárias entre 0-60 anos, os quais foram classificados quanto ao risco à cárie, avaliados em: 1(baixo); 2(médio); 3(alto), fatores contribuintes para o câncer bucal, necessidade e uso de protése dentária e prevalência de doença peridontal. Os dados foram tabulados e submetidos à análise descritiva e Kappa com concordância intra e inter-avaliadores de $100 \%$ e 95\%, respectivamente. Observou-se maior número de indivíduos enquadrados como risco 1 e 2 na zona urbana. Nos fatores contribuintes para o câncer bucal, a exposição ao tabagismo e ao etilismo foi semelhante nas populações analisadas. O uso de prótese, a zona rural (27\%) se destacou em relação à urbana (18,2\%), sendo a faixa etária de 20-59 anos mais cometida por portadores de uso de prótese dentária. A prevalência de alteração na mucosa na mesma faixa etária (20-59 anos) foi fator de destaque entre as duas regiões, tendo a zona rural 6,2\% e a zona urbana 2,8\%. Quanto ao desenolvimento de doença periodontal verificou-se que a zona urbana se destacou em relação a rural, tendo $25,13 \%$ dos indivíduos com a doença ativa. Tais resultados sugerem melhor direcionamento das políticas de saúde bucal e suas estratégias de cuidado, a fim de alcançar acesso ao serviço odontológico e resolutividade baseado nas especificidades de diferentes faixas etárias e localizações.
\end{abstract}

Palavras-chave: Saúde bucal; Epidemiologia; Políticas públicas.

\section{Abstract}

Conducting epidemiological surveys is an important tool for understanding the prevalence of oral diseases and, thus, being able to guide the planning, execution and evaluation of health actions in a population. Thus, a cross-sectional study was carried out with 13.706 people, living in the rural and urban areas, aged 0-60 years, who were classified 
according to the risk of caries, evaluated in: 1(low); 2 (medium), 3 (high), contributing factors for oral cancer, need and use of dental prosthesis and prevalence of periodontal diseases. The data were tabulated and submitted to descriptive and Kappa analysis with intra and inter-rater agreement of $100 \%$ and $95 \%$, respectively. There was a greater number of individuals classified as risk 1 and 2 in the urban area. In the contributing factors for oral cancer exposure to smoking and alcohol consumption was similar in the populations analyzed. The use of prostheses, the rural area (27\%) stood out in relation to the urban area (18.2\%), with the age group of 20-59 years being most committed by patients with the use of dental prosthesis. The prevalence of changes in the mucosa in the same group (20-59 years) was a prominent factor between the two regions, with the rural area $6.2 \%$ and the urban area $2.8 \%$. As for the development of periodontal disease it was found that the urban area stood out in relation to the rural area, with $25.13 \%$ of individuals with active disease. Such results suggest better targeting of oral health policies and their care strategies, in order to achieve access to the dental service and resolution based on the specificities of different age groups and locations.

Keywords: Oral health; Epidemiology; Public policies.

\section{Resumen}

La realización de encuestas epidemiológicas es una herramienta importante para comprender la prevalencia de las enfermedades bucodentales y, así, poder orientar la planificación, ejecución y evaluación de las acciones de salud en una población. Así, se realizó un estudio transversal con 13.706 personas, residentes en el área rural y urbana, de 0 hacia 60 años, que fueron clasificados según el riesgo de cariesl evaluados en: 1 (bajo); 2 (medio); 3 (alto); factores que contribuyen al cáncer oral, la necesidad y el uso de prótesis dentales y la prevalencia de la enfermedad periodontal. Los datos se tabularon y se sometieron a análisis descriptivo y Kappa con un acuerdo intra e interevaluadores del $100 \%$ y el $95 \%$, respectivamente. Hubo un mayor número de individuos clasificados como riesgo 1 y 2 en el área urbana. En los factores que contribuyen al cáncer oral, la exposición al tabaquismo y al consumo de alcohol fue similar en las poblaciones analizadas. En el uso de prótesis, se destacó el área rural (27\%) en relación al área urbana (18,2\%), siendo el grupo de edad de 20 hacia 59 años el más comprometido por los pacientes con el uso de prótesis dentales. La prevalencia de cambios en la mucosa en el mismo grupo de edad (20-59 años) fue un factor prominente entre los dos regiones, con el área rural 6,2\% y el área urbana 2,8\%. En cuanto al desarrollo de la enfermedad periodontal, se encontró que el área urbana se destacó en relación al área rural, con un 25,13\% de individuos con enfermedad activa. Dichos resultados sugieren una mejor focalización de las políticas de salud bucal y sus estrategias de atención, con el fin de lograr el acceso al servicio odontológico y la resolución en función de las especificidades de los diferentes grupos de edad y ubicaciones.

Palabras clave: Salud bucal; Epidemiología; Políticas públicas.

\section{Introdução}

Cárie dentária, doenças periodontais e câncer bucal são considerados importantes problemas de saúde pública no Brasil e no mundo (Kassebaum et al. 2014). No Brasil, cerca de 50\% da população foi relatada com cárie dentária e $99 \%$ com doença periodontal. Portanto, ambas as condições representam as principais causas de perda dentária, além de levarem a um impacto negativo na qualidade de vida da população, devido à redução da capacidade funcional, da autoestima e das relações sociais (Brasil, 2010). Em 2010, uma pesquisa nacional revelou que o Brasil havia melhorado significativamente seus registros e se apresentava como um país com baixa prevalência de cárie (CPOD = 2,07) (Brasil, 2011). Da mesma forma, o levantamento nacional brasileiro demonstrou que as ações governamentais locais afetaram positivamente a prevalência da doença periodontal. Embora consideremos esses resultados animadores, as características regionais devem ser analisadas com cautela, a fim de orientar ações futuras que visem aprimorar as estratégias locais de prestação dos melhores serviços e decisões preventivas às comunidades (Brasil, 2018a).

O câncer bucal é outro evento relevante que deve ser considerado para o estabelecimento de estratégias preventivas eficazes em saúde pública. No Brasil, o Instituto Nacional do Câncer (INCA) estimou 14,7 mil novos casos de câncer bucal, 11,2 mil em homens e 3,5 mil em mulheres (Brasil, 2018a). O uso do tabaco tem sido proposto como um dos fatores de risco mais importantes para o câncer bucal, seguido pelo uso do álcool. Infelizmente, a maioria dos casos é diagnosticado em estágios tardios, contribuindo para altas taxas de mortalidade e morbidade. Assim, programas de rastreamento têm sido propostos como medida de prevenção de baixo custo para identificar indivíduos nos estágios iniciais da doença e melhorar a sobrevida por apresentarem altos escores de acurácia (Santos et al. 2011; Leite et al. 2021). 
No presente estudo, objetivamos definir a prevalência de cárie dentária, doença periodontal e fatores / manifestações de câncer bucal em uma população urbana e rural de uma região Nordeste do Brasil (Penedo, Alagoas, Brasil). Antecipamos que os resultados do presente estudo fornecerão a base para o estabelecimento de estratégias eficazes de prevenção da saúde bucal e, portanto, impactarão positivamente na qualidade de vida da comunidade.

\section{Metodologia}

Foi realizado estudo observacional transversal de natureza quantitativa, de base populacional na cidade de Penedo, região Sul do Estado de Alagoas, Penedo, realizado em 2017. Os estudos populacionais foram classificados em ( $\mathrm{n}=13.706): 1$. Faixa etária com foco no ciclo de vida do indivíduo [0- 2 anos (bebês), 2-9 anos (crianças), 10-19 anos (adolescentes), 20-59 anos (adultos) e idosos com mais de 60 anos]; e 2. região de residência [rural ou urbana] (Brasil, 2018a). As variáveis do estudo foram: 1. Risco de cárie dentária; 2. Câncer oral; e 3. Doença periodontal. O presente estudo foi realizado de acordo com o preconizado pela resolução 196 de 10/10/1996 do Conselho Nacional de Saúde do Ministério da Saúde após submissão e aprovação pelo Comitê de Ética Institucional (CAAE: 68611617.9.0000.5374). Um formulário de consentimento informado (CIF) foi obtido de cada sujeito inscrito e dos responsáveis legais de cada uma das crianças envolvidas no estudo primário. O cálculo do tamanho amostral foi realizado considerando poder de $99 \%$ e alfa $=0,05$, um número total da população de Penedo (64.292) (IBGE, 2017) e o tamanho mínimo da amostra foi de 659 indivíduos. Este estudo de base populacional incluiu 13.706 indivíduos, representando $21 \%$ da população total. A população total residente em áreas urbanas e rurais é composta por $48.992(76,2 \%)$ e 15.300 (23,8\%) pessoas, respectivamente. Na pesquisa uma amostra de $21 \%$ dos indivíduos em cada área de pesquisa foram incluídos, 10.444 ingressantes para a área urbana e 3.262 para a área rural. A amostra em cada faixa etária foi composta por 2.000 a 2.700 (19\% a 26\%) dos participantes da área urbana, e de 620 a 850 (de 19\% a 26\%) dos participantes da área rural, distribuídos entre homens e mulheres, conforme recomendado pela OMS, (Brasil, 2018a).

Participaram do estudo nove equipes de trabalho calibradas, compostas por cirurgião-dentista, agente comunitário de saúde (ACS) e auxiliar de saúde bucal (OHA). Cada equipe ficou responsável por uma microárea. A calibração foi realizada em três etapas: 1. Treinamento, no qual foram apresentados os índices de risco e parâmetros e instrumentos que foram utilizados na pesquisa, 2. Examinadores e anotadores foram treinados por meio da realização de exames odontológicos em crianças de 5 a 9 anos e adultos 20- 59 anos, com seis crianças e seis adultos para treinamento e 10 de cada faixa etária para calibração. Foi realizado exame duplamente em cada grupo de 10 indivíduos, porém, o examinados não fora informado a respeito do exame a fim de não interferir no resultado da reprodução do estudo e 3. Reunião para discussão das dúvidas encontradas, bem como visando padronizar os critérios empregados nos exames. Um examinador de padrão ouro conduziu os processos de treinamento e calibração das equipes para chegar a um acordo geral. Foi alcançado um nível aceitável de estatísticas de concordância entre avaliadores (kappa $=0,85$. Os dados foram coletados em formulário padronizado apresentado pela Secretaria de Estado da Saúde de Alagoas, e de acordo com parâmetros previamente estabelecidos (Brasil, 2018a). Os indivíduos que não estavam em casa no momento da visita e que optassem em não participar do estudo foram excluídos do estudo. Após a execução do inquérito epidemiológico, as equipes de saúde bucal consolidaram os dados coletados em formulário específico, que posteriormente foram armazenados no banco de dados da Secretaria Municipal de Saúde. Com a finalidade de realizar o presente estudo, foram utilizados como variáveis numéricas e percentuais.

Em relação aos códigos e critérios descritos no Quadro 1, resumidamente, o risco de cárie dentária foi avaliado da seguinte forma: 1 = baixo; 2 = médio; 3 = alto; enquanto a doença periodontal foi analisada pela doença gengival e bolsa periodontal. A doença gengival foi verificada a partir da utilização de uma variável ramificada, demonstrando ausência ou presença de sangramento, por se tratar de um método de uso simples, não passível de interpretação subjetiva, e amplamente utilizado em inquéritos e rastreamento de grupos populacionais (Brasil, 2018a). 
Para a análise da presença de bolsas periodontais, foi utilizado o Índice Periodontal Comunitário (IPC), onde foi realizado com uma sonda específica, denominada sonda CPI (Golgran, Blumenau, SC, Brasil). A sonda periodontal foi inserida sem forte pressão no sulco gengival ou na bolsa periodontal e levemente inclinada e direcionada ao longo eixo do dente, acompanhando a inclinação radicular. Embora a OMS não tenha estimado tempo preciso para a visualização de sangramento à sondagem, o mesmo foi observado na faixa de 10 a 30 segundos. A boca dos participantes foi dividida em sextantes definidos pelos dentes 18-14, 13-23, 24-28, 38-34, 33-43 e 44-48. Notando-se dois ou mais dentes sem indicação de exodontia (por furca prejudicada ou mobilidade), foi um pré-requisito para o exame daquele sextante. Sem que isso fosse observado, o sextante fora cancelado. Também não foi considerado o terceiro molar na contagem dos dentes do sextante. Os dentes indicadores para cada sextante até 19 anos foram os dentes 16,11,26, 36, 31 e 46, e em crianças até 6 anos apenas a doença gengival foi analisada. A partir de 20 anos de idade, os dentes analisados foram 17, 16, 11, 26, 27, 37, 36, 31, 46 e 47. Caso nenhum deles estivesse presente, os dentes remanescentes do sextante eram examinados, desconsiderando os terceiros molares. Os participantes que apresentavam sangramento gengival e bolsas maiores que $4 \mathrm{~mm}$ foram considerados como portadores de doença periodontal clinicamente visível, e todos aqueles que apresentavam bolsas periodontais abaixo de $4 \mathrm{~mm}$ e sem sangramento gengival foram considerados sem doença periodontal (Brasil, 2018a).

Em relação ao câncer bucal, a busca ativa incluiu fatores de risco para o câncer bucal, como tabagismo e etilismo, próteses dentárias, alterações da mucosa (exceto na faixa etária de 0 a 2 e 2 a 9 anos). Neste estudo, fumantes ativos foram considerados participantes que consumiram mais de 100 cigarros na vida (Brasil, 2018b; Azevedo et. al 2020). Para serem considerados como "tendo o hábito de beber", Participantes que relataram consumir pelo menos um copo de bebida alcoólica no período de 24horas (Neves, Texeira, Ferreira, 2015).

Quadro 1 - Parâmetros empregados para a averiguação do risco de cárie dentária e Busca ativa de câncer bucal e doença periodontal.

\begin{tabular}{|c|c|c|c|c|}
\hline & CÓDIGO 1 & CODIGO 2 & CODIGO 3 & \\
\hline $\begin{array}{l}\text { Exame objetivo } \\
\text { da boca - lesões } \\
\text { de cárie }\end{array}$ & $\begin{array}{l}\text { * Inexistência de lesão } \\
\text { cariosa e sem: biofilme, } \\
\text { gengivite e/ou mancha } \\
\text { branca ativa. } \\
\text { * Dente restaurado, } \\
\text { porém sem: gengivite } \\
\text { e/ou mancha branca } \\
\text { ativa }\end{array}$ & $\begin{array}{l}\text { *uma ou mais lesões cariosas } \\
\text { crônicas porém sem biofilme, } \\
\text { gengivite e/ ou mancha branca } \\
\text { ativa. } \\
\text { *Inexistência de lesões de cárie, } \\
\text { com: presença de biofilme, de } \\
\text { gengivite e/ou mancha branca }\end{array}$ & $\begin{array}{l}\text { *Uma ou mais cavidades em } \\
\text { situação de lesão de cárie ativa } \\
\text { *presença de dor e ou abcesso }\end{array}$ & \\
\hline \multirow{2}{*}{$\begin{array}{l}\text { Busca ativa ao } \\
\text { câncer bucal }\end{array}$} & Fuma & Bebe & $\begin{array}{l}\text { Lesões por Prótese mal } \\
\text { adaptada }\end{array}$ & Lesões em mucosa \\
\hline & $\begin{array}{c}\text { Cachimbo } \\
\text { Cigarro } \\
\text { Mascar fumo }\end{array}$ & Pelo mais de uma vez por semana & & Por vícios ou hábitos \\
\hline $\begin{array}{l}\text { Doença } \\
\text { periodontal }\end{array}$ & $\begin{array}{l}\text { Presença de doença } \\
\text { periodontal visível } \\
\text { clinicamente com } \\
\text { bolsas periodontais }\end{array}$ & $\begin{array}{l}\text { Presença de sangramento } \\
\text { gengival. }\end{array}$ & Presença de cálculo. & $\begin{array}{ll}\text { Sem } & \text { doença } \\
\text { periodontal } & \text { visível } \\
\text { clinicamente } & \end{array}$ \\
\hline
\end{tabular}

Fonte: Autores. 


\section{Resultados}

Em geral, a área urbana apresentou maior prevalência de risco de cárie (40\% e 33\%), para baixo e médio risco, respectivamente) do que a área rural (35\% e $25 \%$, para baixo e médio risco, respectivamente), enquanto os $40 \%$ da população rural foi caracterizada como risco 3 em comparação com $27 \%$ da área urbana. A análise de dados demonstrou ainda que 35\% da população na área rural tinha risco 1 , ou seja, uma população livre de cárie sem evidência clínica de gengivite e lesões ativas de manchas brancas (Quadro 2).

Analisando as faixas etárias, na faixa de 0 a 2 anos, não foi observada diferença entre as áreas urbana e rural, independentemente do risco de cárie (Quadro 2). Porém, a prevalência de lesões cariosas foi diferente nas partes do município estudadas, destacando-se a zona urbana relacionada ao baixo risco, com exceção das faixas de 10-19 anos e 20-59 anos, onde apresentaram maior proporção de indivíduos com risco 2 (17,2\% e 20\%) quando comparados à área rural $(3,2$ e 4,4\%) respectivamente, significando que, nessas faixas etárias 2,655 dos indivíduos analisados na área urbana apresentam pelo menos uma cavidade com lesão crônica de cárie, ou presença de biofilme visível, gengivite e / ou lesão de mancha branca, conforme Quadro 2.

Quadro 2 - Risco de cárie dentária em diferentes faixas etárias de indivíduos pertencentes às Unidades de saúde do ESF localizadas na zona urbana (10.444) e zona rural (3.262).

\begin{tabular}{|c|c|c|c|c|c|c|c|c|c|c|c|c|}
\hline \multirow{3}{*}{$\begin{array}{c}\text { Faixa etária } \\
0-2 \text { anos }\end{array}$} & \multicolumn{4}{|c|}{ Risco 1} & \multicolumn{4}{|c|}{ Risco 2} & \multicolumn{4}{|c|}{ Risco 3} \\
\hline & \multicolumn{2}{|c|}{ Urbana } & \multicolumn{2}{|c|}{ Rural } & \multicolumn{2}{|c|}{ Urbana } & \multicolumn{2}{|c|}{ Rural } & \multicolumn{2}{|c|}{ Urbana } & \multicolumn{2}{|c|}{ Rural } \\
\hline & 245 & $2 \%$ & 98 & $3 \%$ & 12 & $0,1 \%$ & 09 & $0,08 \%$ & 26 & $0,2 \%$ & 05 & $0.1 \%$ \\
\hline 2-9 anos & 635 & $6 \%$ & 181 & $5,6 \%$ & 265 & $2,5 \%$ & 112 & $1 \%$ & 204 & $2 \%$ & 180 & $5,5 \%$ \\
\hline 10-19 anos & 832 & $8 \%$ & 150 & $4,6 \%$ & 561 & $17,2 \%$ & 107 & $3,2 \%$ & 443 & $4,2 \%$ & 249 & $7,6 \%$ \\
\hline $25-59$ anos & 656 & $6 \%$ & 421 & $13 \%$ & 2.094 & $20 \%$ & 465 & $4,4 \%$ & 1376 & $13 \%$ & 722 & $22 \%$ \\
\hline Acima de 60 anos & 834 & $8 \%$ & 150 & $6 \%$ & 536 & $5,1 \%$ & 117 & $1, \%$ & 725 & $7 \%$ & 146 & $5 \%$ \\
\hline Total & \multicolumn{2}{|l|}{4.202} & \multicolumn{2}{|c|}{1.150} & \multicolumn{2}{|c|}{3.468} & \multicolumn{2}{|c|}{810} & \multicolumn{2}{|l|}{2.774} & \multicolumn{2}{|c|}{1302} \\
\hline Percentual & \multicolumn{2}{|l|}{$40 \%$} & \multicolumn{2}{|c|}{$35 \%$} & \multicolumn{2}{|c|}{$33 \%$} & \multicolumn{2}{|c|}{$25 \%$} & \multicolumn{2}{|c|}{$27 \%$} & \multicolumn{2}{|c|}{$40 \%$} \\
\hline
\end{tabular}

Fonte: Autores.

Quando analisamos, de modo geral, a presença de doença periodontal clinicamente visível, constatamos que $25,2 \%$ e 23, 6\% dos indivíduos analisados na zona urbana e zona rural respectivamente, apresentavam a doença ativa, sem diferença estatística entre as diferentes áreas analisadas. Observando as faixas etárias percebes-e que a zona rural se destacou em relação a zona urbana, na faixa etária de 20-59 anos, onde foi observado um percentual de 16,2\% de pessoas com doença periodontal ativa, quando comparado a 13,6\% dos acometidos pela doença na zona urbana. Porém, na faixa etária de 10 a 19 anos, a zona urbana concentrava mais do que o dobro $(2,6 \%)$ de portadores de doenças periodontais, quando comparada à zona rural $(1,2 \%)$, conforme mostra o Quadro 3. 
Quadro 3 - Necessidade de tratamento periodontal em diferentes faixas etárias de indivíduos pertencentes as Unidades de saúde do ESF localizadas na zona urbana (10.444) e zona rural (3.262).

\begin{tabular}{|c|l|l|c|c|c|c|c|c|}
\hline \multirow{2}{*}{ Faixa etária } & \multicolumn{3}{|c|}{ Doença periodontal presente } & \multicolumn{3}{c|}{ Doença periodontal ausente } \\
\cline { 2 - 9 } & \multicolumn{2}{|c|}{ Urbana } & \multicolumn{2}{c|}{ Rural } & \multicolumn{2}{c|}{ Urbana } & \multicolumn{2}{c|}{ Rural } \\
\hline $0-2$ anos & 11 & $0,1 \%$ & 01 & $0,03 \%$ & 347 & $3,3 \%$ & 105 & $3,2 \%$ \\
\hline $2-9$ anos & 64 & $0,6 \%$ & 10 & $0,3 \%$ & 1168 & $11,1 \%$ & 512 & $15,7 \%$ \\
\hline $10-19$ anos & 273 & $2.6 \%$ & 42 & $1,28 \%$ & 1.550 & $14,8 \%$ & 401 & $12,3 \%$ \\
\hline $25-59$ anos & 1.428 & $13,6 \%$ & 531 & $16,2 \%$ & 3.623 & $34,6 \%$ & 1.213 & $37 \%$ \\
\hline Acima de 60 anos & 860 & $8,23 \%$ & 186 & $5,7 \%$ & 1.120 & $10,7 \%$ & 261 & $8, \%$ \\
\hline Total & 2.636 & $25,13 \%$ & 770 & $23,51 \%$ & 7.808 & $74,5 \%$ & 2.492 & $76,2 \%$ \\
\hline
\end{tabular}

Fonte: Autores.

Quanto à busca ativa por câncer de boca, de maneira geral, observou-se exposição semelhante ao tabagismo e ao consumo de álcool para as populações rural e urbana (Quadro 3). Porém, isso não ocorreu no que se refere ao uso de próteses, destacando-se a zona rural (27\%) em relação à zona urbana (18\%), sendo a faixa etária de 20 a 59 anos a mais comprometida por pacientes com necessidade de uso de prótese. A prevalência de alteração na mucosa na mesma faixa etária (20-59 anos) também foi um fator de destaque entre as duas regiões, com a área rural $(6,2 \%)$ mais que o dobro do que a urbana $(2,8 \%)$. Isso sugere a necessidade de intensificar e estender as ações de controle dos fatores de risco à população mais jovem, conforme mostra o Quadro 4.

Quadro 4 - Fatores de risco de Câncer Bucal em diferentes faixas etárias de indivíduos pertencentes as Unidades de ESF da zona urbana e zona rural.

\begin{tabular}{|l|l|l|l|l|l|l|l|l|}
\hline \multirow{3}{*}{ Faixa etária } & \multicolumn{5}{|c|}{ Zona Urbana } & \multicolumn{5}{c|}{ Zona Rural } \\
\cline { 2 - 9 } & \multicolumn{3}{|c|}{10.444} & \multicolumn{5}{c|}{3.262} \\
\cline { 2 - 9 } & Fuma & Bebe & $\begin{array}{l}\text { Usa } \\
\text { prótese }\end{array}$ & $\begin{array}{l}\text { Alteração } \\
\text { Mucosa }\end{array}$ & Fuma & Bebe & Usa prótese & $\begin{array}{l}\text { Alteração } \\
\text { mucosa }\end{array}$ \\
\hline $2-9$ anos & 04 & 0 & 0 & 02 & 0 & 0 & 0 & 0 \\
\hline $10-19$ & 220 & 32 & 19 & 16 & 25 & 48 & 03 & 05 \\
\hline $20-59$ & 298 & 759 & 1020 & 162 & 150 & 138 & 702 & 109 \\
\hline ACIMA DE 60 & 331 & 138 & 862 & 115 & 80 & 60 & 182 & 90 \\
\hline Total & 853 & 949 & 1.901 & 295 & 265 & 216 & 827 & 269 \\
\hline Percentual & $8,2 \%$ & $9,1 \%$ & $18,2 \%$ & $2,8 \%$ & $7,8 \%$ & $7,5 \%$ & $27 \%$ & $6.2 \%$ \\
\hline
\end{tabular}

Fonte: Autores.

\section{Discussão}

Em saúde bucal, a epidemiologia se define como os estudos que trazem informações básicas sobre a saúde bucal e/ou acerca das necessidades de tratamento odontológico de uma população em tempo e locais definidos. Os objetivos destes estudos visam observar os problemas odontológicos e monitorar possíveis modificações nos níveis e padrões das doenças durante o passar dos anos (Neves et al., 2019). Neste cenário, a presente pesquisa mostra-se como estratégia essencial para transformação da realidade local. Entretanto, percebe-se uma resistência por parte dos profissionais e gestores em utilizar esta ferramenta quando se fala em decisões de saúde. Além disso, no processo de trabalho das Estratégias em Saúde Bucal (ESB) à 
atenção ainda está fortemente voltada para o modelo biomédico, não havendo muitas vezes, uma interlocução com diagnóstico situacional do território ao qual a estratégia está inserida. Sendo as tomadas de decisões baseadas no cotidiano da clínica odontológica, vinculado a ações clínicas, a partir de ações preventivas e restauradoras direcionadas aos escolares, sendo então inaptas à respostas das necessidades daquela população, conforme ressaltado por Scherer \& Scherer 2015; Scherer et al., 2018.

Alguns autores destacaram a importância dos dados epidemiológicos para custearo planejamento de ações em saúde bucal da população, para que a intervenção resulte em impacto positivo necessário ao controle das doenças bucais, promovendo saúde e melhorando a qualidade de vida, com a análise fiel da realidade daquela área que está sendo estudada e possibilitando comparar dados referentes à situação local com as metas propostas pela OMS e outros estudos nacionais (Chaves, 2007; Brasil, 2009, Moysés, 2013; Brasil; 2018). Em concordância, as equipes envolvidas na presente investigação utilizaram da interpretação dos dados coletados para a elaboração do plano de intervenção baseado na realidade local de distintas regiões de um mesmo município. Dessa forma, o levantamento epidemiológico realizado no município de PenedoAL, demonstrou a necessidade do conhecimento profundo da população usuária, como elemento primordial que possa vir a desvincular da gestão baseada na oferta, inserindo um olhar diferenciado, baseado nas especificidades das duas regiões, zonal rural e zona urbana.

No que diz respeito à prevalência de cárie, estudos realizados nos anos 80 demonstraram maior prevalência de cárie dentária na população rural em comparação à população urbana (Marques, et al. 1986). Em 1990, observou-se redução de cárie no meio rural, mas estudos realizados em 2004, puderam comprovar que a saúde bucal em populações rurais ainda é bastante deficitária (Abreu, et al., 2004). Os dados observados no último levantamento epidemiológico nacional do SB Brasil 2010, revelaram modificações no perfil epidemiológico das doenças bucais apontadas, colocando o Brasil entre os países com baixa prevalência de cárie, CPO-D 2,07 (Brasil, 2011). Entretanto, apesar de os resultados apresentados fossem satisfatórios nacionalmente, ressaltam a atenção para desigualdades regionais na prevalência e gravidade da doença cárie que foram preponderantes, indicando primordial idade de políticas voltadas para a equidade na atenção. Da mesma forma, no município de Penedo-AL, a população rural apresentou maior prevalência da doença cárie, risco 3 em todas as faixas etárias maiores de dois anos, mostrando resultados indesejados em relação ao esperado, realçando a necessidade de maiores cuidados e intervenções voltadas à promover uma política de saúde bucal voltada as especificidades local, tais como dificuldade de acesso, cultura local, promoção e prevenção, trabalhando o usuário e a família em sua integralidade, rompendo com o modelo fragmentado, baseado na demanda que visa o favorecimento de populações privilegiadas com desvantagem às mais vulneráveis.

Resultados semelhantes ao do município de Penedo- AL, foram encontrados no município de Campinas que, objetivando planejar ações relativas à saúde bucal do Centro de Saúde de Barão Geraldo, foi feito levantamento epidemiológico no período de 2003, em duas unidades de ensino público, situadas nas áreas urbana e rural, com a finalidade de observação de prevalência de lesões de cárie e possibilitar o dimensionamento de necessidades de tratamento e o demanda por assistência odontológica. Observou-se disparidade entre a prevalência de cárie nas duas populações estudadas, sendo o CPO-D aos 12 anos para a escola rural igual a 3,0 e o da escola urbana igual a 0,87, levando o gestor municipal de saúde a instalar um consultório odontológico na área rural, pois a falta destes equipamentos estava conduzindo à dificuldade no acesso da população ali adscrita à atenção odontológica adequada.. O mesmo foi observado num estudo realizado no Município de Pindoba-AL em 2016 em escolas de localização na zona rural, baseado no índice de risco de cárie dental (risco 1, 2, 3) e doença periodontal, necessidade de instalação de prótese dentária. Visando elaborar um plano de intervenção para prevenção e tratamento odontológico para os alunos que apresentarem qualquer problema de saúde bucal. Observou-se uma porcentagem de 26,6\% até 52,17\% do número de escolares com risco 3 (Omena, 2016).

Observou-se também no analise do perfil epidemiológico, que apesar da zona urbana ter se destacado em relação aos 
baixos índices para doença cárie, a mesma se destacou nas faixas etárias de 10- 19 anos e 20 a 59 anos apresentando ou maiores índices de risco 2 , em relação a população rural, merecendo uma política de saúde bucal voltada ao perfil desses usuários. É importante ressaltar que nessa faixa etária ocorre uma maior probabilidade para desenvolvimento de doenças crônicas como diabetes, tuberculose, AIDS e outras que podem apresentar manifestações bucais. Esses problemas apesar de ocorrer em outras faixas etárias adquirem grande relevância nessas faixas etárias (Brasil, 2008). Além disso, esse resultado pode ter relação com a disponibilidade de tempo desses usuários para frequentar as unidades de saúde durante o período diurno. O que implica na importância de desenvolvimento de estratégias que possibilitem horários alternativos voltados ao atendimento e promoção em saúde bucal (Azevedo et. al (2020).

Em relação à prevalência da doença periodontal, observam-se vários fatores de risco para o desenvolvimento da doença periodontal, como ausência ou dificuldades no controle do biofilme dentário, fatores socioeconômicos e culturais, larga exposição a fatores ambientais deletérios, como tabagismo, o fato de ser portador de doenças cônicas como diabetes e exposição ao estresse crônico e condições de imunossupressão (Brasil, 2018).

No último SB Brasil (2010), os dados relativos às doenças periodontais indicam um crescimento destas situações com o aumento da idade da população, apontam para um aumento desses problemas com o avançar da idade, atingindo um número de apenas $1,8 \%$ de idosos (65 a 74 anos) livres de doenças periodontais. Naquele estudo, as doenças gengivais serem demonstradas com pouca expressão na população idosa se mostra de forma contraditória devido menor número de dentes presentes (Brasil, 2011).

Inquérito epidemiológico realizado no município de Pindoba-AL, mostrou que 10\% das crianças de 7 a 12 anos, em localidades rurais, apresentavam doença periodontal ativa (Omena, 2016). Diferentemente do que foi encontrado, o presente estudo mostrou uma prevalência de $24,9 \%$ dos indivíduos analisados, que apresentavam a doença ativa, sem diferença estatística entre as diferentes zonas analisadas. No entanto, a faixa etária de 20 a 59 anos apresentou maior envolvimento de doença periodontal clinicamente visível quando comparada às demais faixas etárias.

Esses resultados comprovam a deficiência ou falta de controle do biofilme devido às deficiências funcionais, já que problemas sensoriais e articulares, como a artrite, dificultam a compreensão dos resultados clínicos odontológicos, assim como a comunicação de suas necessidades. e preocupações com as alternativas de atenção odontológica, assim como, impossibilitando o autocuidado bucal efetivo (Spackman; Bauer, 2016).

Os resultados sugerem ainda que existe problema com relação ao acesso aos serviços odontológicos, sendo eles são uma escolha arbitrária, desvinculada das ações da atenção primária em saúde, estando direcionados à baixa renda e à falta de atendimento regular, observado no reduzido número de dentes remanescentes resultantes de cultura cirúrgica mutiladora.

Quanto à busca ativa do câncer bucal, observou-se que com relação à exposição aos fatores de risco voltados ao tabagismo e etilismo, as duas populações urbana e rural apresentaram-se proporcionalmente iguais. Um dado preocupante, pois, como já comprovado cientificamente, a etiologia do câncer bucal é uma reunião de fatores carcinógenos que podem conduzir ao surgimento da doença, dentre eles, os de origem extrínseca, tais como o tabagismo e o etilismo, que são responsáveis por aproximadamente $75 \%$ dos casos nos países desenvolvidos e que quando associados, atuam em sinergia, agregando risco para o desenvolvimento do câncer de boca (Zain, 2001; Wünsch-Filho, 2002; Bagan \&Scully, 2009; Leite et al. 2021). Isso demonstra a necessidade de estratégia de saúde bucal ativa e vigilante para esses dois fatores, e com ênfase em políticas de saúde bucal voltadas ao autoexame.

França et al. (2010; Lima, Dwyer, 2020) exaltam o papel da equipe de saúde Bucal no aconselhamento de pacientes e para diagnóstico de câncer bucal, identificando, os fatores de risco como o tabagismo, que podem levar ao ressecamento da mucosa oral, aumento da queratinização epitelial, além da produção do trauma térmico- mecânico; o etilismo por sua vez, que por contato crônico com a mucosa jugal age como solvente diminuindo a velocidade de reação com o organismo e produzindo 
danos irreparáveis à estrutura celular. Ressaltam ainda atenção a imunodeficiência, enfatizando-se o vírus HPV. Alertando acerca da importância da realização de biopsia de lesões pré cancerizáveis somadas a tais agentes etiológicos tendem a aumentar as chances de sofrerem modificações, transformando-se em carcinoma in situ.

No concernente ao uso de prótese mal adaptada e lesões de tecido mole a zona rural (29\%, $6 \%$ respectivamente) se destacou em relação a urbana (19\%, 3\% respectivamente) na faixa etária de 20-59 anos, apresentando maior predisposição ao desenvolvimento de lesões pré-cancerígenas. $\mathrm{O}$ trauma crônico da mucosa oral causado por mordiscamentos, irritação por próteses mal adaptadas, traumas causados por lesões por escovação dental e exposição da mucosa a uma borda aguda de cárie dentária, fraturas dentais ou ação de qualquer outro irritante externo, pode levar a evolução para malignidade (Mateus, 2008; Brasil, 2009; Piemont et al., 2010). Sendo assim, as equipes de saúde bucal são os atores principais envolvidos no processo de diagnóstico de etiologia multifatorial.

Outro fato observado em Penedo -AL, foi que $90 \%$ da população da zona rural e em $52 \%$ da urbana aos usuários não tinham esclarecimento em relação aos fatores de risco para o câncer bucal e negligenciavam sinais e sintomas. O que leva a reafirmar o relatado por Quirino et al. 2006; Brasil 2018; Damasceno, Cruz \& Barros 2021) que atribui as políticas públicas e Estratégia de saúde bucal, o desenvolvimento de estratégias de fácil compreensão e acessibilidade para os usuários voltadas a promoção, prevenção e diagnóstico precoce e possível intervenção para câncer bucal e ou lesões pré cancerizáveis.

\section{Considerações Finais}

Após o levantamento epidemiológico realizado constatou-se que o risco da doença cárie sofreu variações em relação a faixa etária e localização. Sendo os indivíduos da zona rural e faixa etária de 25-59 anos da zona urbana mais propensos aos agravos da doença cárie. Sugerindo uma atenção maior quanto a promoção da saúde, prevenção de agravos, tratamento e reabilitação em saúde bucal, baseado no perfil e especificidades populacional.

Por outro lado, para o câncer bucal, ambas as regiões mostraram considerável exposição aos variados fatores de risco, principalmente, a tabaco e álcool. Isso pode significar a deficiência de conscientização, de maneira geral, da população em relação ao câncer de boca e que as intervenções sobre o assunto devem ser intensificadas.

Por fim, observa-se que os levantamentos epidemiológicos devem ser inseridos no processo de trabalho das equipes de saúde bucal e serem vistos como indispensáveis para planejamento, programas de intervenção e avaliação no setor público, permitindo uma proposta de uma política local de saúde bucal bem delimitada.

Sugere-se que os futuros levantamento epidemiológicos realizados no município, possam contemplar os povos especiais como quilombolas, indígenas, pescadores, não analisados nesse estudo. De forma, a discutir e ampliar as estratégias no processo de trabalho das equipes de saúde bucal, ofertando maior acessibilidade e resolutividade ao sistema única de saúde, fazendo-se valer os princípios básicos da universalidade, equidade e integralidade.

\section{Referências}

Abreu, M. H. N. G., Pordeus I. A. \& Modena C. M. (2004). Cárie dentária entre escolares do meio rural de Itaúna (MG), Brasil. Rev Panam Salud Publica, 16(5), 334-344

Antunes, J. L. F. \& Peres, M. A. (2013). Epidemiologia em Saúde Bucal. Guanabara Koogan.

Azevedo R. C., Pereira A. C. A., Medeiros, Y. L., Feyo, V. B., Luiz, K. A., Esteves, T. C., Chaves, M. G. A. M. \& Fabri, G. M. C. (2020). Necessidades de atendimento odontológico em pacientes com câncer durante a pandemia de COVID-19 - uma revisão. Research, Society and Development. 9(12).

Bagan. J. V. \& Scully, C. (2008) Recent advances in Oral Oncology; squamous cell carcinoma aetiopathogenesis and experimental studies. Oral Oncology, $45(6) ; 45-48$.

Brasil, Ministério da Saúde. (2009). Instituto Nacional do Câncer. Estimativa 2010: Incidência de Câncer no Brasil/Instituto Nacional de Câncer. Brasil, Ministério da Saúde. (2018b). Instituto Nacional do Câncer. Estimativa 2018: Incidência de Câncer no Brasil/Instituto Nacional de Câncer. INCA. 130 p. 
Brasil, Ministério da Saúde. (2018 a a). Secretaria de Atenção à Saúde. Departamento de Atenção Básica. A saúde bucal no Sistema Único de Saúde [recurso eletrônico] / Ministério da Saúde, Secretaria de Atenção à Saúde, Departamento de Atenção Básica. - Brasília.

Brasil, Ministério da Saúde. (2010). Secretaria de Atenção à saúde. Departamento de Atenção Básica. Coordenação Geral de Saúde Bucal. Pesquisa Nacional de Saúde Bucal. Brasília.

Brasil, Ministério da Saúde. (2011). Secretaria de Atenção à Saúde. Departamento de Atenção Básica. Coordenação Geral de Saúde Bucal. Pesquisa Nacional de Saúde Bucal SB Brasil 2010: resultados principais. Brasília.

Brasil, Ministério da Saúde. (2008). Secretaria de Atenção à Saúde. Departamento de Atenção Básica. Saúde Bucal. Série A. Normas e Manuais TécnicosCaderno de Atenção Básic., nº17. $1^{\text {a }}$ ed.

Brasil, Ministério da Saúde. (2009). Secretaria de Políticas da Saúde. Departamento de Atenção Básica. Área Técnica de Saúde Bucal. Projeto SB 2010: condições de saúde bucal da população brasileira no ano 2010. Brasília: Ministério da Saúde.

Campinas, Secretaria Municipal de Saúde. (2003). Relatório do Levantamento Epidemiológico em Saúde Bucal. Módulo Village Paidéia do Centro de Saúde de Barão Geraldo. Campinas.

Chaves, S. C. L. \& Silva, L. M. V. (2007). Atenção em saúde bucal e a descentralização de saúde no Brasil: Estudo de dois casos exemplares do Estado da Bahia. Caderno de Saúde Pública, 23(5), 1119-1131.

Cimard, A. C. B. S. \& Fernandes, A. P. S. (2009). Câncer Bucal: A prática e a realidade clínica dos cirurgiões dentistas de Santa Catarina. RFO. 2(14), 99-104.

Damasceno, K. S. M., Cruz, D. N. \& Barros, C. G (2021). Acessibilidade aos serviços odontológicos no SUS: revisão da literatura. Research, Society and Development. 10 (3).

França, D. C. C., Pinto, M. M. O., Monteiro, A. D., Silva, A. A. L. S., Zina, O., \& Lima, G. S. (2010). Programa de diagnóstico e prevenção de câncer de boca: Uma estratégia simples e eficaz. Rev odont. Bras Central. 19 (49): 159-161.

Gjermo, P., Rosing, C.K. \& Susin, C. (2002). Periodontal diseases in central and south America.; 29(1), $70-78$.

IBGE, Instituto Brasileiro de Geografia e 2017 . https://cidades.ibge.gov.br/content/index.php?option=com_content\&view=articl\&id=20607\&Itemid=7572.

INCA, Instituto Nacional do Câncer. Estimativa do Câncer 2016/2017. http://www.inca.gov.br/estimativa/2016/estimativa-2016-v11.pdf.

Kassebaum, N. J., Bernabé, E., Dahiya, M., Bhandari, B., Murray, C. J. \& Marcenes, W. (2014). Global burden of severe periodontitis in 1990-2010: a systematic review and meta-regression. J Dent Res. 93(11):1045-1053.

Leite, R. B., Marinho, A. C. O., Costa, B. L., Laranjeira, M. B. V., Araújo, K. D. T. \& Cavalcanti, A. F. M. (2021) A influência da associação de tabaco e álcool no câncer bucal: revisão de literatura. J. Bras. 57: 1-5. Rio de Janeiro.

Lima, F. L. T. \& O’Dwyer, G. (2020). Políticas de Prevenção e Controle do Câncer Bucal à luz da Teoria da Estruturação de Giddens. Ciênc. saúde coletiva. $25(8)$

Marques, D. E., Rink, M. C. M., Loureiro, R. M. T. \& Silva, V. C. (1986). Levantamento epidemiológico de cárie dentária na zona rural de Uberlândia, Minas Gerais: contribuição para um modelo de programa de saúde bucal. Rev Cent Ciênc Bioméd Univ Fed Uberlândia. 2(1), 33-38.

Mateus, F. O. (2008). Câncer bucal no Brasil: Revisão de literatura. Faculdade de Medicina. Curso de Especialização em Saúde Pública. Universidade Federal do Rio Grande do Sul.

Moysés, S,J. (2013). Saúde Coletiva, Políticas, Epidemiologia da Saúde Bucal e Redes de Atenção Odontológica. Artes Médicas.

Neves, K. C., Teixeira, M. L. O. \& Ferreira, M. A. (2015). Fatores e motivação para o consumo de bebidas alcoólicas na adolescência. Revista de Enfermagem. Jun. 19 (2): 286-291.

Omena, B. D. (2016). Levantamento Epidemiológico da Necessidade de Tratamento Odontológico das escolas da zona rural do município de Pindoba- AL. Universidade Federal de Santa Catarina.

OMS, Organização Mundial da Saúde. (2013). Levantamentos em saúde bucal: Métodos Básicos. (5 ed.).

Piemonte, E. D., Lazos, J. P. \& Brunotto, M. (2010). Relationship between chronic trauma of the oral mucosa, oral potentially malignant disorders and oral cancer. Jornal of Oral Pathology \& Medicine. 39(7), 513-517.

Prado, N. F. \& Passarelli, D. H. C. (2009). Uma nova visão sobre prevenção do câncer bucal no consultório odontológico. Rev.Odont.Univer. SP. 21(1), 29-85.

Quirino, M. R. S., Gomes, F. C, Marcondes M. S. \& Baducci, I. (2006). Avaliação do conhecimento sobre o câncer de boca entre os participantes de campanha para prevenção e diagnóstico precoce da doença em Taubaté- SP. Rev. Odontol UNES. 35(4), 327-333.

Santos, I. V., Alves, T. D. B., Falcão, M. M. L. \& Freitas, V. S. (2011). O papel do cirurgião-dentista em relação ao câncer de boca. Odontol. Clín.-Cient. 10(3):207-210.

Scherer, C. I. \& Scherer, M. D. A. (2015). Avanços e desafios da saúde bucal após uma década de Programa Brasil Sorridente. Rev. Saúde Pública, 49, 98.

Scherer, C. I., Scherer, M. D. A., Chaves, S. C. L. \& Menezes, E. L. C. (2018). O trabalho em saúde bucal na Estratégia Saúde da Família: uma difícil integração? 
Research, Society and Development, v. 10, n. 6, e42110615368, 2021

(CC BY 4.0) | ISSN 2525-3409 | DOI: http://dx.doi.org/10.33448/rsd-v10i6.15368

Saúde debate. .42(2):233-246.

SESAL, Secretaria Estadual de Saúde de Alagoas. (2009). Gerência de núcleo de saúde bucal. Manual de atenção básica em saúde bucal do Estado de Alagoas. p. 8-9.

Spackman, S. S. \& Bauer, J. G. (2016). Correlation of the Index of Activities of Daily Living (Index of ADOH) with the Functional Independence Measure (FIM) in Older Adults. J Gerontol Geriatr Res. Mar, 5:2.

Vasconcelos, E. M. \& Fratucci, V. B. (2014) Práticas de Saúde Bucal. http://www.unasus.unifesp.br/biblioteca_virtual/esf/2/unidades_conteudos/uni dade15o/unid ade15o.pdf.

Wünsch-Filh, V. (2002). The epidemiology of oral and pharynx cancer in Brazil. Oral Oncol. 38, 737-746.

Zain, R. B. (2001). Cultural and dietary risk factors of oral cancer and precancer a brief overview. Oral oncology. 37, 205-210. 